\title{
Les Aleocharinae (Insecta, Coleoptera, Staphylinidae) de Nouvelle-Calédonie, avec la description de nouvelles espèces
}

Roberto PACE

Via Vittorio Veneto, 13,

I-37032 Monteforte d'Alpone (Verona) (Italie)

pace.ent@tiscali.it

MOTS CLÉS

Insecta,

Coleoptera,

Staphylinidae,

Aleocharinae,

Nouvelle-Calédonie, espèces nouvelles.

KEY WORDS

Insecta,

Coleoptera,

Staphylinidae,

Aleocharinae,

New Caledonia, new species.
Pace R. 2009. - Les Aleocharinae (Insecta, Coleoptera, Staphylinidae) de Nouvelle-Calédonie, avec la description de nouvelles espèces. Zoosystema 31 (4): 749-758.

\section{RÉSUMÉ}

Sept espèces nouvelles d'Aleocharinae appartenant aux tribus des Homalotini (Lomaglossina giachinoi $\mathrm{n}$. sp., L. amieuensis $\mathrm{n}$. sp., Neidosphena miricornis $\mathrm{n} . \mathrm{sp}$., Nemoedia amieuensis n. sp.), Athetini (Aglaogaster giachinoi $\mathrm{n}$. sp.) et Thamiareini (Thamiaraea kanakn. sp., Mimacrotona giachinoi n. sp.), sont décrites et illustrées. Le mâle de Nemoedia solitaria Pace, 1991, jusqu'alors inconnu, est illustré. Avec le présent travail, 125 espèces d'Aleocharinae sont désormais connues de Nouvelle-Calédonie, pour la plupart endémiques.

\section{ABSTRACT}

Aleocharinae (Insecta, Coleoptera, Staphylinidae) from New Caledonia with description of new species.

Seven new species of Aleocharinae belonging to the tribes Homalotini (Lomaglossina giachinoi n. sp., L. amieuensis n. sp., Neidosphena miricornis n. sp., Nemoedia amieuensis n. sp.), Athetini (Aglaogaster giachinoi n. sp.) and Thamiareini (Thamiaraea kanak n. sp., Mimacrotona giachinoi n. sp.) are described and illustrated. The formerly unknown male of Nemoedia solitaria Pace, 1991 is illustrated. With the present paper, 125 species are known from New Caledonia, most of them endemic 


\section{INTRODUCTION}

La sous-famille des Aleocharinae Fleming, 1821 comporte de nombreux genres et espèces, et occupe toutes les régions zoogéographiques. En NouvelleCalédonie, 32 genres étaient jusqu’à présent répertoriés, représentés par 118 espèces, presque toutes endémiques (Pace 1981, 1989, 1991, 1997).

Sept nouvelles espèces sont décrites ici, appartenant à trois tribus différentes d'Aleocharinae: Lomaglossina giachinoi n. sp., L. amieuensis n. sp., Neidosphena miricornis n. sp. et Nemoedia amieuensis n. sp. (Homalotini), Aglaogaster giachinoi n. sp. (Athetini) et Thamiaraea kanak n. sp. et Mimacrotona giachinoi n. sp. (Thamiareini), ce qui porte à 125 le nombre d'espèces d'Aleocharinae désormais connues de ce territoire.

La connaissance des espèces néocalédoniennes d'Aleocharinae est cependant encore loin d'être achevée. En effet, même si les travaux précédents étaient basés non seulement sur les séries typiques de Fauvel $(1878,1889)$ et de Scheerpeltz (1966), mais aussi sur le matériel ancien disponible dans les muséums et sur des récoltes récentes, les recherches se sont communément concentrées autour de Nouméa et de La Foa. Les autres zones de l'île, notamment la Province Nord, n'ont jamais été prospectées.

\section{MATÉRIEL ET MÉTHODES}

Le matériel étudié a été récolté en Nouvelle-Calédonie par le Dr Pier Mauro Giachino, qui me l'a confié pour étude.

L'étude taxonomique des espèces d'Aleocharinae nécessite entre autre l'examen des parties buccales, de la formule tarsale, de l'édéage, de la spermathèque.

Les exemplaires étudiés ici ont donc été disséqués et les structures buccales et génitales incluses dans du baume du Canada, sur de petits rectangles transparents en matière plastique associés à chaque spécimen. Les structures génitales ont été étudiées à l'aide d'un microscope permettant des agrandissements jusqu'à $450 \times$ et dessinées au moyen d'un oculaire quadrillé. Les mesures ont été prises avec un micromètre oculaire. Les caractères de l'habitus ont été observés avec un microscope stéréoscopique jusqu’à $100 \times$. Toutes les illustrations ont été réalisées par l'auteur.

Les holotypes, les paratypes et la totalité du matériel déterminé sont déposés au Muséum national d'Histoire naturelle, Paris (MNHN).

\section{SYSTÉMATIQUE}

Famille STAPHYLINIDAE Latreille, 1802

Sous-famille AleOCHARINAE Fleming, 1821

Tribu Pronomaeini Mulsant \& Rey, 1873

Genre Myllaena Erichson, 1837

Myllaena numeensis Pace, 1991

Myllaena numeensis Pace, 1991: 83.

Matériel eXAminé. - Nouvelle-Calédonie. Mt Humboldt for., mousse, 1330 m, 15.II.2006, P. M. Giachino leg., 1 ○ (MNHN).

Distribution. - Espèce connue seulement des Mts Koghis, nouvelle pour le Mt Humboldt.

Tribu GyrophaENini Kraatz, 1856

Genre Sternotropa Cameron, 1920

Sternotropa monoincisa Pace, 1997

Sternotropa monoincisa Pace, 1997: 263.

Matériel eXaminé. - Nouvelle-Calédonie. La Foa, Col d'Amieu, 500 m, 20.II.2006, P. M. Giachino leg., 2 우 (MNHN).

DistRibution. - Espèce connue seulement de la Rivière Bleue, nouvelle pour le Col d'Amieu.

Tribu Homalotini Heer, 1839

Genre Aphelusa Pace, 1981

Aphelusa caledonica (Fauvel, 1878)

Sipalia caledonica Fauvel, 1878: 591.

Leptusa (s.s.) caledonica - Bernhauer \& Scheerpeltz 1926: 554

Aphelusa caledonica - Pace 1981: 161; 1989: 23; 1991 : 114. 
Matériel eXaminé. - Nouvelle-Calédonie. La Foa, Col d'Amieu, 500 m, 20.II.2006, P. M. Giachino leg., $10^{\text {" }}(\mathrm{MNHN})$.

DisTRIBUTION. - Espèce connue de la Rivière Bleue, de la Forêt de Thi Hanna, des Mts Koghis, du Col de Mouiranga et du Mt. Panié. Nouvelle signalisation au Col d'Amieu.

\section{Genre Lomaglossina Pace, 1991}

\section{Lomaglossina (Lomaglossina) domina Pace, 1997}

Lomaglossina domina Pace, 1997: 267.

Matériel examiné. - Nouvelle-Calédonie. La Foa, Col d'Amieu, 500 m, 20.II.2006, P. M. Giachino leg., 1 우 (MNHN).

DisTRibution. - Espèce connue seulement de la Rivière Bleue, nouvelle pour le Col d'Amieu.

\section{Lomaglossina (Lomaglossina) kanak Pace, 1991}

Lomaglossina (Lomaglossina) kanak Pace, 1991: 122.

Matériel examiné. - Nouvelle-Calédonie. La Foa Pocquereux, 50 m, 10-24.II.2006, P. M. Giachino leg., 2 ơ $^{\pi}, 3$ 우 (MNHN).

Distribution. - Espèce connue du Col d'Amieu et du Col des Roussettes.

\section{Lomaglossina (Lomaglossina) parens Pace, 1991}

Lomaglossina (Lomaglossina) parens Pace, 1991: 122.

Matériel examiné. - Nouvelle-Calédonie, La Foa Pocquereux, 50 m, 10-24.II.2006, P. M. Giachino leg. 12 exemplaires (MNHN).

Distribution. - Espèce connue uniquement du Col d'Amieu.

\section{Lomaglossina (Lomaglossina) giachinoi $\mathrm{n} . \mathrm{sp}$.} (Fig. 1A-E)

Holotype. - Nouvelle-Calédonie. La Foa, Col d'Amieu, 500 m, 20.II.2006, P. M. Giachino leg., ơ (MNHN).

PARATYPes. - Même provenance que l'holotype, 45 exemplaires (MNHN).
ÉTymologie. - La nouvelle espèce est dédiée à son récolteur, le Dr Pier Mauro Giachino.

Diagnose. - Vu de profil, l'édéage de la nouvelle espèce est dentelé dans sa partie distale ventrale, celle-ci étant très étroite vue de profil. Aucune espèce connue ne possède l'ensemble de ces caractères.

\section{DESCRIPTION}

Longueur 2,5-2,7 mm. Corps brillant et jaune-rougeâtre, tête jaune-brun, élytres bruns, une large tache brune médiane sur le quatrième urotergite libre et à la base du cinquième, antennes brunes avec les deux antennomères basaux et le onzième antennomère jaune-rougeâtres, pattes jaune-rougeâtres. Deuxième antennomère plus court que le premier, le troisième plus court que le deuxième, antennomères 4 à 10 transverses. Yeux aussi longs que les tempes. Réticulation de la tête et du pronotum évidente, celle des élytres forte, celle de l'abdomen transverse et distincte. Ponctuation de la tête dense et superficielle, celle du pronotum fine et aussi dense. Granulation des élytres dense et saillante.

\section{Mâle}

Troisième urotergite libre avec un tubercule médian fort, sixième urotergite libre avec une carène médiane saillante (Fig. 1E). Édéage Figure 1A, B.

\section{Femelle}

Spermathèque Figure 1D.

\section{Lomaglossina (Lomaglossina) amieuensis n. sp.} (Fig. 1F-J)

Holotype. - Nouvelle-Calédonie. La Foa, Col d'Amieu, 500 m, 8-20.II.2006, P. M. Giachino leg., ơ (MNHN).

PARATYPes. - Même provenance que l'holotype, 11 exemplaires (MNHN).

ÉTYMOLOGiE. - Le nom de la nouvelle espèce dérive de celui de la localité type: Col d'Amieu.

DiAGNOSE. - Par la forme de l'édéage, la nouvelle espèce ressemble à $L$. domina Pace, 1997. Elle s'en distingue par le côté préapical ventral de l'édéage dentelé (sans dentelure chez L. domina), par la longueur de l'édéage $(0,33 \mathrm{~mm}$, contre $0,45 \mathrm{~mm}$ chez L. domina) et par la forme de la 
marge postérieure du sixième urotergite libre du mâle (six petites dents marginales chez L. domina). La tête et les élytres sont d'autre part bruns (tête jaune-rougeâtre et élytres partiellement jaune-rougeâtres chez L. domina).

\section{DESCRIPTION}

Longueur 2-2,2 mm. Corps brillant et jaune-rougeâtre, tête, élytres, quatrième urotergite libre et la base du cinquième bruns, antennes brunes avec les deux antennomères basaux et la base du troisième antennomère jaune-rougeâtres, pattes jaune-rougeâtres. Deuxième antennomère plus court que le premier, le troisième bien plus court que le deuxième, les antennomères 4 à 10 transverses. Réticulation de la tête, du pronotum et des élytres évidente et fine, celle de l'abdomen transverse et distincte. Ponctuation de la tête fine et très superficielle, celle du pronotum serrée et presque invisible. Granulation des élytres serrée et saillante, celle de l'abdomen fine, serrée et superficielle.

\section{Mâle}

Édéage Figure 1H, I. Sixième urotergite libre Figure 1J.

\section{Femelle}

Spermathèque Figure 1G.

Genre Neidosphena Pace, 1991

Neidosphena miricornis n. sp.

(Fig. 2A, B)

Holotype. - Nouvelle-Calédonie. Mt Humboldt presso sorgente, 1270 m, 15.II.2006, P. M. Giachino leg., ? (MNHN).

ÉTYMologie. - Le nom de la nouvelle espèce signifie "antennes merveilleuses", en raison de leur belle couleur.

DiAgnOSE. - Par la couleur noir-brune du corps et par les antennomères terminaux jaunes, la nouvelle espèce est différente des espèces connues, qui soit présentent un corps en partie ou principalement jaune-rougeâtre, soit possèdent seulement le onzième antennomère jaune. Cette nouvelle espèce se distingue également par la forme de sa spermathèque. Par son bulbe distal subsphérique la nouvelle espèce ressemble à $N$. newtoni Pace, 1991 et
N. koghicola Pace, 1991, mais ces espèces ont la partie proximale de la spermathèque plus courte que la partie distale.

\section{DESCRIPTION}

Longueur 2,3 mm. Corps brillant et noir-brun, pygidium brun, antennes brunes avec le premier antennomère et les antennomères 6 à 10 jaunes, pattes jaune-rougeâtres. Deuxième antennomère aussi long que le premier, le troisième plus court que le second, les quatrième et cinquième plus longs que larges, les sixième et septième aussi longs que larges, les huitième au dixième transverses. Yeux aussi longs que les tempes. Corps sans réticulation. Ponctuation de la tête serrée, très superficielle et absente sur le front, celle du pronotum et des élytres fine, serrée et évidente. Granulation de l'abdomen évidente, mais présente seulement sur la moitié postérieure des quatrième et cinquième urotergites libres de la femelle.

\section{Femelle}

Spermathèque Figure 2B.

Mâle

Inconnu.

Genre Nemoedia Pace, 1991

Nemoedia solitaria Pace, 1991

(Fig. 2C-E)

Nemoedia solitaria Pace, 1991: 131.

Matériel eXAminé. - Nouvelle-Calédonie. Mt Humboldt for., Mousse, 1330 m, 15.II.2006, P. M. Giachino leg., $10^{\circ "}(\mathrm{MNHN})$.

Distribution. - Espèce connue seulement des Mts Koghis, nouvelle pour le Mt Humbolt.

\section{REMARQue}

Cette espèce n'était jusqu'à présent connue que par une femelle. L'identification du mâle a été possible, non seulement par la couleur du corps et la forme des antennomères, mais aussi par le rapport largeur/ longueur du pronotum de 1,45 identique dans les deux exemplaires. Edéage Figure 2C, D. 

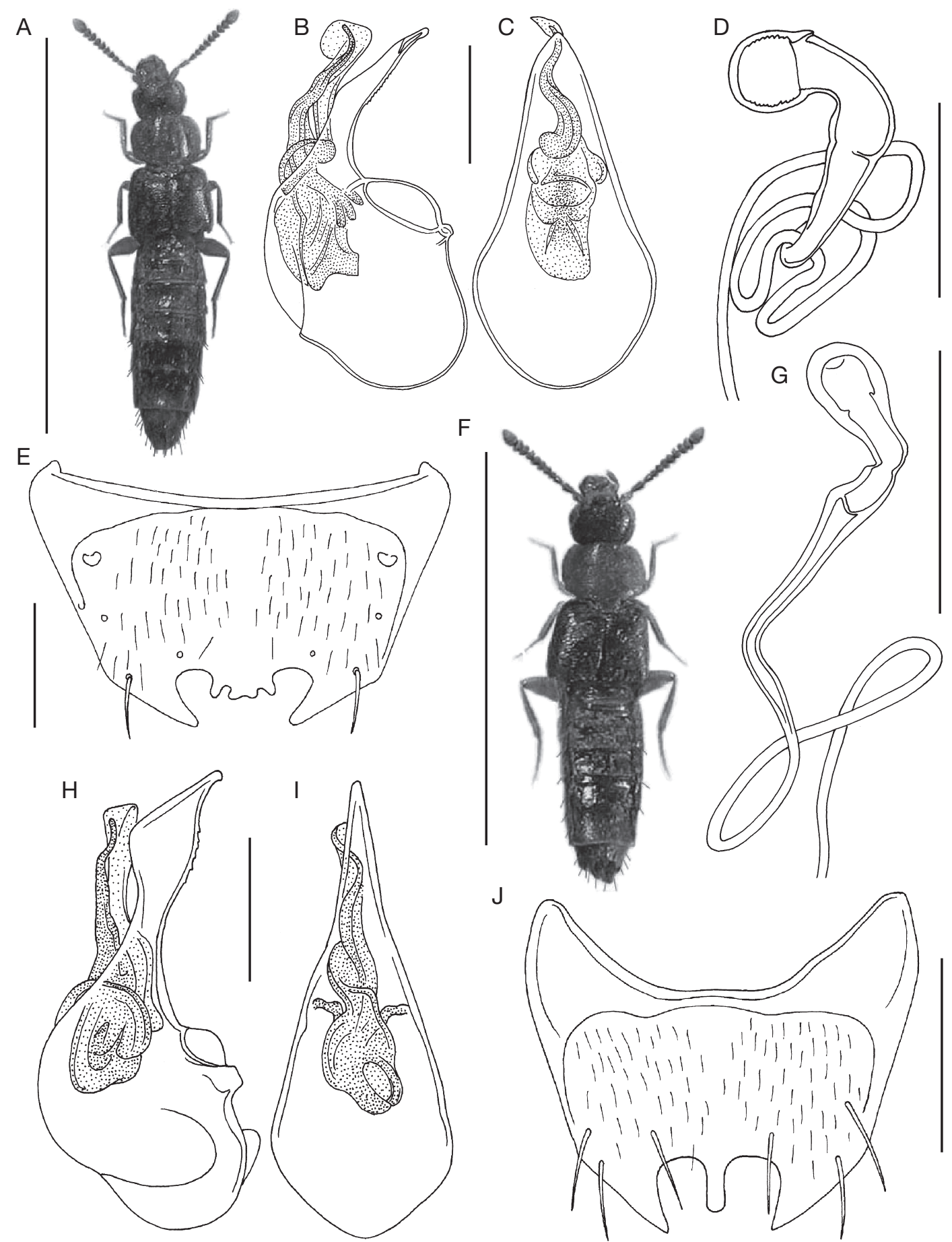

FIG. 1. - Habitus, édéage vu de profil et ventralement, spermathèque et sixième urotergite libre du ơ : A-E, Lomaglossina (Lomaglossina) giachinoi n. sp.; F-J, L. (L.) amieuensis n. sp. Échelles: A, 2,5 mm; B-E, G-J 0,1 mm; F, 2 mm. 
Nemoedia divisa Pace, 1997

Nemoedia divisa Pace, 1997: 270.

Matériel eXAminé. - Nouvelle-Calédonie. La Foa, Col d'Amieu, 500 m, 20.II.2006, P. M. Giachino leg., 3 o $^{7}$ (MNHN).

Distribution. - Espèce jusqu’à présent connue de la Rivière Bleue, nouvellement signalée du Col d'Amieu.

\section{Nemoedia amieuensis n. sp.} (Fig. 2F-J)

Holotype. - Nouvelle-Calédonie. La Foa, Col d'Amieu, 500 m, 8-20.II.2006, P. M. Giachino leg., $\mathrm{o}^{7}(\mathrm{MNHN})$.

PARATYPES. - Même provenance que l'holotype, 10 , 5 우 (MNHN).

ÉTYMOLOGIE. - Le nom de la nouvelle espèce dérive de la localité type: Col d'Amieu.

DiAgnose. - Par la forme de l'édéage et de la spermathèque, la nouvelle espèce ressemble à $N$. neocaledonica Pace, 1991, également décrite du Col d'Amieu. Elle s'en distingue par l'apex de l'édéage court, vu de profil (long chez $N$. neocaledonica), vu du coté ventrale asymétrique et étroit (large, symétrique et avec incision chez $N$. neocaledonica). En outre les antennomères quatre à sept sont fort transverses dans la nouvelle espèce, alors qu'ils sont plus longs que larges chez $N$. neocaledonica.

\section{DESCRIPTION}

Longueur 2,1-2,3 mm. Avant-corps faiblement opaque, abdomen brillant. Corps jaune-rougeâtre, élytres jaune-brun, leur base jaune-rougeâtre. Troisième, quatrième et moitié basale du cinquième urotergite libre bruns. Antennes rougeâtres avec les trois antennomères basaux jaunes et le onzième brun, pattes jaunes. Deuxième antennomère plus court que le premier, le troisième plus court que le deuxième, les antennomères quatre à 10 fortement transverses. Yeux plus longs que les tempes. Réticulation de la tête et de l'abdomen évidente, celle du pronotum et des élytres forte.

\section{Mâle}

Sixième urotergite libre avec une carène médiane saillante et profondément creusé à la moitié de la marge postérieure. Édéage Figure 2G, H.

\section{Femelle}

Spermathèque Figure 2I, sixième urotergite libre Figure 2J.

\section{Tribu Diestotini Mulsant \& Rey, 1872 \\ Genre DiestotaMulsant \& Rey, 1872}

Diestota numeensis (Fauvel, 1889)

Bolitochara numeensis Fauvel, 1889: 280. — Bernhauer \& Scheerpeltz 1926: 566.

Diestota numeensis - Pace 1991: 143.

Matériel examiné. - Nouvelle-Calédonie. La Foa Pocquereux, 50 m, 10-24.II.2006, P. M. Giachino leg., 4 exemplaires (MNHN). — La Foa, Col d'Amieu, $500 \mathrm{~m}$, 20.II.2006, P. M. Giachino leg., 1 ơ, 1 ๆ (MNHN).

Distribution. - Espèce largement répandue en Nouvelle-Calédonie.

Tribu ATHETini Casey, 1910

Genre Aglaogaster Pace, 1985

Aglaogaster caledonicum Pace, 1985

Aglaogaster caledonicum Pace, 1985: 113; 1991: 162.

Matériel eXaminé. - Nouvelle-Calédonie. La Foa, Col d'Amieu, 500 m, 8-20.II.2006, P. M. Giachino leg., 17 exemplaires (MNHN). - La Foa Pocquereux, $50 \mathrm{~m}$, 10-24.II.2006, P. M. Giachino leg., 1 ơ (MNHN).

Distribution. - Espèce largement répandue en Nouvelle-Calédonie, et déjà signalée du Col d'Amieu.

\section{Aglaogaster giachinoi n. sp.}

(Figs 2K, L; 3A-D)

Holotype. - Nouvelle-Calédonie. La Foa Pocquereux, 50 m, 10-24.II.2006, P. M. Giachino leg., O" (MNHN).

PARATYPES. - Même provenance que l'holotype, 8 spécimens (MNHN).

ÉTYMOLOGIE. - La nouvelle espèce est dédiée à son récolteur, le Dr Pier Mauro Giachino.

Diagnose. - Par la forme de la ligule (Fig. 2L) et de l'édéage, la nouvelle espèce ressemble à $A$. caledonicum Pace, 


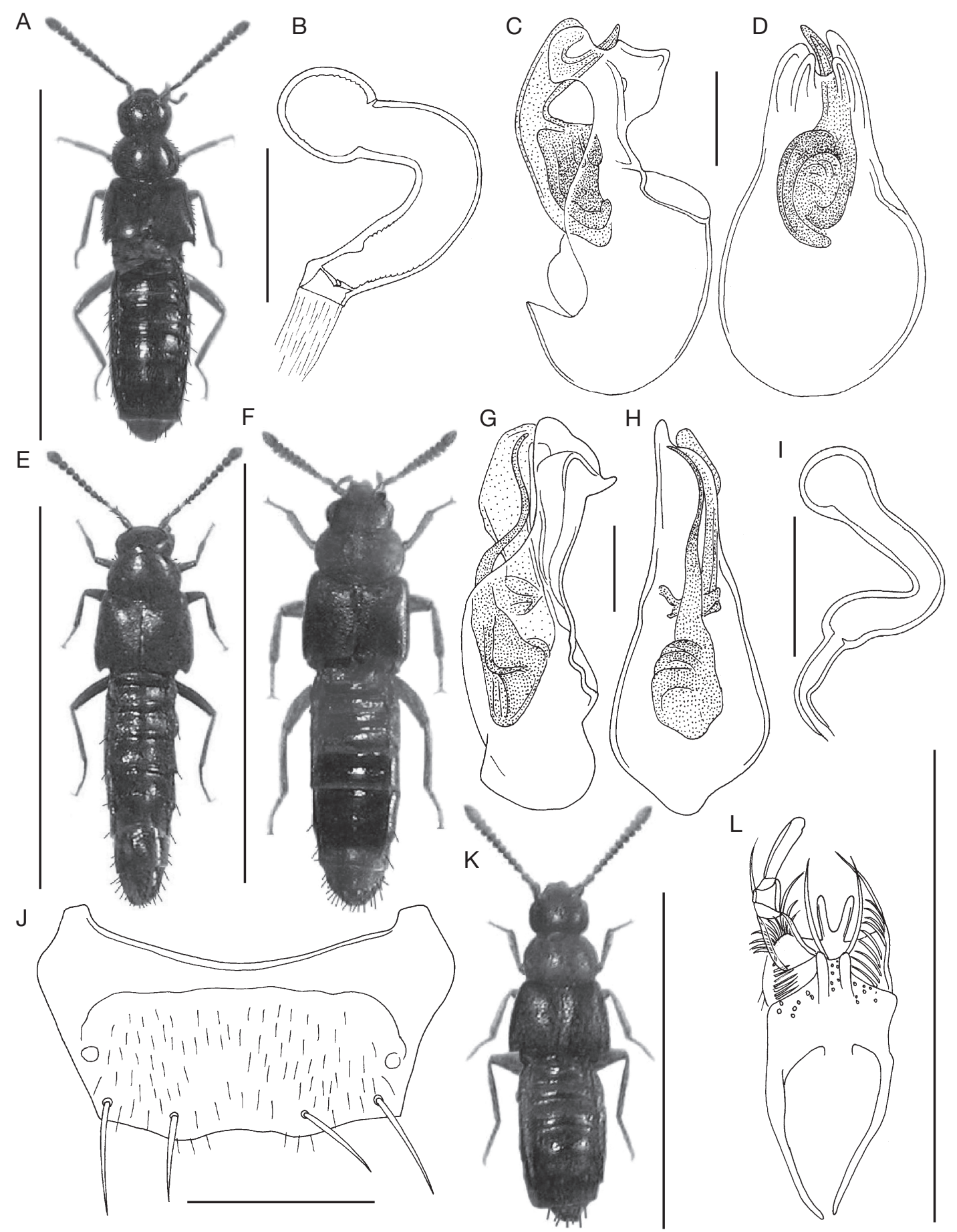

FIG. 2. - Habitus, spermathèque, édéage vu de profil et ventralement, sixième urotergite libre de la $\$$ et labium avec palpe labial: A, B, Neidosphena miricornis n. sp.; C-E, Nemoedia solitaria Pace, 1991; F-J, Nemoedia amieuensis n. sp.; K, L, Aglaogaster giachinoi n. sp. Échelles: A, 2,3 mm; B-D, G-J, L, 0,1 mm; E, 2,2 mm; F, 2,1 mm; K, 1,3 mm. 
1985 mais cette dernière espèce a des élytres, mesurés de l'angle huméral à l'angle extérieur postérieur, plus courts que le pronotum. La spermathèque de la nouvelle espèce possède, en outre, une partie proximale plus étroite que la portion intermédiaire, tandis que chez $A$. caledonicum la partie proximale est bien plus large que la partie intermédiaire.

\section{DESCRIPTION}

Longueur 1,3 mm. Corps brillant et jaune-rougeâtre, élytres et quatrième et cinquième urotergites libres bruns, antennes brunes avec les trois antennomères basaux jaune-rougeâtres et les deux terminaux jaunes, pattes jaunes. Deuxième antennomère aussi long que le premier, le troisième plus court que le second, les antennomères quatre à 10 transverses. Yeux plus courts que les tempes. Réticulation de la tête et des élytres évidente, celle du pronotum peu distincte et celle de l'abdomen superficielle. Ponctuation de la tête indistincte. Granulation du pronotum fine, serrée et saillante, celle des élytres superficielle et celle de l'abdomen fine et distincte.

Mâle

Édéage Figure 3A, B.

Femelle

Spermathèque Figure 3D.

Tribu Thamiaraeini Fenyes, 1921

Genre Thamiaraea Thomson, 1858

\section{Thamiaraea kanak n. sp.}

(Fig. 3E, F)

Holotype. - Nouvelle-Calédonie. La Foa Pocquereux, 50 m, 10-24.II.2006, P. M. Giachino leg., ○ (MNHN).

ÉTYMOLOGIE. - La nouvelle espèce est dédiée à la population Kanak de la Nouvelle-Calédonie.

DIAGNOSE. - Par la forme de la spermathèque la nouvelle espèce ressemble à T. scapularis sailendra Pace, 1991 de Java. Elle s'en distingue par le quatrième antennomère aussi long que large (plus long que large chez T. scapularis sailendra) et par sa spermathèque mince (trapue chez T. scapularis sailendra), dont le bulbe distal est à peine plus large que la partie intermédiaire; chez T. scapularis sailendra le bulbe distal de la spermathèque est bien plus large que la partie intermédiaire.

\section{DESCRIPTION}

Longueur 3,1 mm. Pronotum opaque, tête et élytres faiblement brillants. Corps jaune-rougeâtre, les deux tiers postérieurs des élytres et des troisième et quatrième urotergites libres bruns, antennes brunes avec les trois antennomères basaux jaune-rougeâtres, pattes jaunes, des reflets iridescents sur les urotergites libres quatre et cinq. Deuxième antennomère plus court que le premier, le troisième aussi long que le deuxième, le quatrième aussi long que large, les antennomères cinq à dix transverses. Yeux plus longs que les tempes. Réticulation de la tête fine et forte, celle du pronotum vigoureuse, celle des élytres évidente; abdomen sans réticulation. Ponctuation de la tête, du pronotum et des élytres serrée et très superficielle, absente sur une étroite bande médiane longitudinale de la tête. Des granules le long de la marge postérieure des urotergites libres. Un point setigère de chaque côté des urotergites libres.

\section{Mâle}

Inconnu.

\section{Femelle}

Spermathèque Figure 3F.

Genre Xenastenoglossa Pace, 1991

Xenastenoglossa pusilla Pace, 1991

Xenastenoglossa pusilla Pace, 1991: 154.

Matériel eXaminé. - Nouvelle-Calédonie. La Foa, Col d'Amieu, 500 m, 8-20.II.2006, P. M. Giachino leg., $20^{7} 0^{7}$ (MNHN).

Distribution. - Espèce connue des Mts Koghis, nouvellement signalée du Col d'Amieu.

\section{Xenastenoglossa trinotata Pace, 1991}

Xenastenoglossa trinotata Pace, 1991: 154.

Matériel eXAminé. - Nouvelle-Calédonie. La Foa, Col d'Amieu, 500 m, 8-20.II.2006, P. M. Giachino leg., 1 ㅇ (MNHN).

DistRibution. - Espèce connue des alentours de Nouméa et de La Foa, nouvellement signalée du Col d'Amieu. 


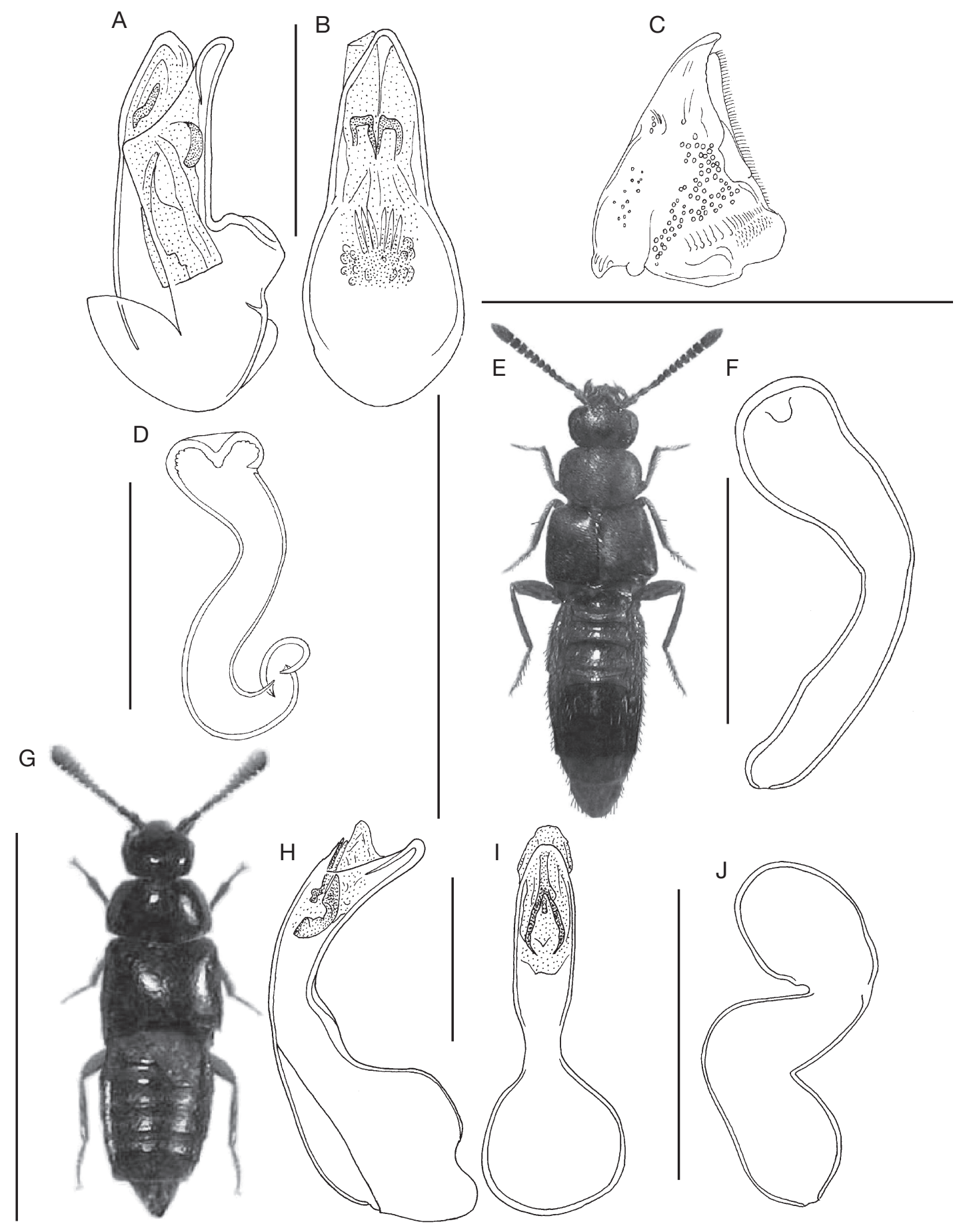

FIG. 3. - Édéage vu de profil et ventralement, mandibule droite vue ventralement et spermathèque: A-D, Aglaogaster giachinoi n. sp.; E, F, Thamiaraea kanak n. sp.; G-J, Mimacrotona giachinoi n. sp. Échelles: A-D, F, H-J, 0,1 mm; E, 3,1 mm; G, 1,6 mm. 
Genre Mimacrotona Cameron, 1920

\section{Mimacrotona giachinoi n. sp.}

(Fig. 3G-J)

Holotype. - Nouvelle-Calédonie. La Foa, Col d'Amieu, 500 m, 8-20.II.2006, P. M. Giachino leg., ơ (MNHN).

PARATYPe. - Même provenance que l'holotype, 1 \% $(\mathrm{MNHN})$

ÉTYMOLOGIE. - La nouvelle espèce est dédiée à son récolteur, le Dr Pier Mauro Giachino.

DiAGNOSE. - La nouvelle espèce présente un abdomen entièrement jaune-rougeâtre et l'antennomère 11 de la même couleur que les antennomères précédents. Lédéage est arqué du côté ventral. Par ces caractères la nouvelle espèce ressemble à $M$. neocaledonica Pace, 1991 décrite de Hyenghène. Elle s'en distingue par un édéage plus développé, long de $0,21 \mathrm{~mm}(0,17 \mathrm{~mm}$ chez $M$. neocaledonica) avec un bulbe basal plus volumineux, et par son pronotum moins transverse (rapport largeur/ longueur de 1,47, contre 1,71 chez $M$. neocaledonica).

\section{DESCRIPTION}

Longueur 1,6 mm. Corps brillant. Tête et pronotum bruns, élytres brun-rougeâtre, abdomen jaune-rougeâtre, antennes jaunes avec les antennomères deux à quatre bruns, pattes jaunes. Deuxième antennomère plus court que le premier, le troisième plus court que le deuxième, les antennomères quatre à dix transverses. Yeux plus longs que les tempes. Réticulation absente sur le corps. Ponctuation de la tête fine et peu évidente, celle des élytres serrée et superficielle. Granulation du pronotum fine et saillante, celle de l'abdomen serrée et très superficielle.

\section{Mâle}

Édéage Figure 3H, I.

\section{Femelle}

Spermathèque Figure 3J.

\section{Remerciements}

Je remercie très cordialement le Dr Pier Mauro Giachino de Turin, spécialiste connu de Catopidae et Carabidae, pour m'avoir confié l'étude de ses récoltes d'Aleocharinae. Je remercie vivement le Prof. J.-C. Lecoq de Mirepeix (France), spécialiste reconnu de Staphylinidae, pour avoir lu et corrigé le style de ma traduction en langue française du présent travail.

\section{RÉFÉRENCES}

Bernhauer M. \& ScheErPeltz O. 1926. - Staphylinidae VI, in Junk W. \& SCHENKLING S., Coleopterorum Catalogus, pars 82. Junk, Berlin: 499-988.

Fauvel A. 1878. - Les staphylinides de l'Australie et de la Polynésie. Annali del Museo Civico di Storia Naturale di Genova 13: 465-598.

Fauvel A. 1889b. - Les Coléoptères de la NouvelleCalédonie et dépendances avec descriptions, notes et synonymies nouvelles - Staphylinides. Revue d'Entomologie 18: 277-282.

PACE R. 1981. - Descrizione di un nuovo genere per Leptusa caledonica (Fauvel). Bollettino della Società entomologica italiana 113: 159-162.

PACE R. 1985. - Aleocharinae attere della regione australiana e neozelandese raccolte dal Prof. Franz (Coleoptera, Staphylinidae). Fragmenta Entomologica 18: 105-114.

PACE R. 1989. - Monografia del genere Leptusa Kraatz. Memorie del Museo civico di Storia naturale di Verona 8: 1-307.

PACE R. 1991. - La sottofamiglia Aleocharinae della Nuova Caledonia (Coleoptera Staphylinidae). Memorie della Società entomologica italiana 70: 79-170.

PACE R. 1997. - Nuove Aleocharinae della Nuova Caledonia (Coleoptera Staphylinidae), in NAJT J. \& Matile L. (eds), Zoologia Neocaledonica, Volume 4. Mémoires du Muséum national d'Histoire naturelle 171: 257-282.

SCheERPeltz O. 1966. - Ergebnisse der Österreichischen Neukaledonien-Expedition 1965. Annalen des Naturhistorischen Museums in Wien 63: 393-418.

Soumis le 10 mars 2008; accepté le 8 octobre 2008. 